\title{
Nódulo pulmonar solitario
}

Un nódulo pulmonar solitario (NPS) puede ser el modo de presentación de procesos benignos o malignos, constituyendo un desafío diagnóstico.

La definición del nódulo pulmonar es radiológica y se refiere a la opacidad pulmonar solitaria en la radiografia de tórax, rodeada de parénquima normal y no mayor a $3 \mathrm{~cm}$.

En el caso de que corresponda a un cáncer de pulmon el NPS es, por definición, una lesión de estadio I y potencialmente curable.

El desafío consiste en lograr resecar todos los nódulos malignos, evitando estudios diagnósticos invasivos y costosos en los pacientes con nódulos benignos.

Según han mostrado las series radiológicas realizadas con el objetivo de rastrear el cáncer de pulmon, la prevalencia de NPS en la población general es 1 en 1000 (se aclara que no está recomendado hacer rastreo de cáncer pulmonar en los individuos asintomáticos). La probabilidad global de malignidad varia entre el 4 y el $10 \%$.

Con la tomografía computada se ha logrado una mejor selección de los candidatos a la biopsia, siendo malignos un 60 a un $80 \%$ de los NPS resecados.

Los factores que influyen en el riesgo de malignidad de un NPS son: el tamaño del nódulo,

la edad del paciente,

la historia de tabaquismo y

la historia previa de cáncer.

El $80 \%$ de los nódulos mayores a $3 \mathrm{~cm}$ son malignios y sólo el $20 \%$, de los menores de $2 \mathrm{~cm}$.

En las series quirúrgicas, un $65 \%$ de los pacientes mayores de 50 años y un 35\% de los menores a 50 años tuvieron cáncer en los nódulos extirpados. Globalmente, la posibilidad de cáncer en los menores de 35 años es muy baja.

El 80\% de los pacientes con un NPS y un cáncer previo tiene enfermedad maligna en dicho nódulo (metástasis o un segundo tumor primario).

Presentación clínica y radiológica del nódulo pulmonar solitario El NPS suele acompañarse de pocos síntomas asociados, o carecer de los mismos según algunas definiciones estrictas. Algunos síntomas pueden ser: tos, broncorrea o hemóptisis. En este último caso resulta más correcto hablar de un paciente con hemóptisis que tiene en la radiografía de tórax una lesión pulmonar.

Las causas de NPS pueden dividirse en malignas y benignas y se enumeran en el cuadro 1.

Cuadro 1: Causas de nódulo pulmonar solitario

\begin{tabular}{|c|c|c|c|}
\hline \multicolumn{2}{|c|}{ Causas malignas } & \multicolumn{2}{|c|}{ Causas benignas } \\
\hline $\begin{array}{l}\text { Carcinoma } \\
\text { broncogénico } \\
(70 \%)\end{array}$ & Adenocarcinoma & $\begin{array}{l}\text { Granuloma infeccioso } \\
(80 \%)\end{array}$ & Tuberculosis \\
\hline & $\begin{array}{l}\text { Carcinoma } \\
\text { epidermoide }\end{array}$ & & Histoplasmosis \\
\hline & Células grandes & \multicolumn{2}{|l|}{ Hamartoma $(5 \%)$} \\
\hline & Células pequeñas & \multicolumn{2}{|l|}{ Absceso } \\
\hline $\begin{array}{l}\text { Metastaticos } \\
\text { (10 a } 30 \%)\end{array}$ & Cáncer de mama & \multicolumn{2}{|c|}{ Granulomatosis de Wegener } \\
\hline & Cáncer de colon & \multicolumn{2}{|c|}{ Nódulos reumatoides } \\
\hline & Cáncer de riñón & \multicolumn{2}{|c|}{ Malformacion arteriovenosa } \\
\hline & $\begin{array}{l}\text { Cáncer de cabeza } \\
\text { y cuello }\end{array}$ & \multicolumn{2}{|c|}{ Infarto pulmonar } \\
\hline & Sarcomas & \multicolumn{2}{|l|}{ Quiste broncogénico } \\
\hline & Tumor germinal & \multicolumn{2}{|c|}{ Lipoma, fibroma, amiloidoma } \\
\hline \multirow[t]{2}{*}{$\begin{array}{l}\text { Pulmonares } \\
\text { no bronco- } \\
\text { génicos }\end{array}$} & $\begin{array}{l}\text { Carcinoide } \\
\text { bronquial* }\end{array}$ & Parasitosis & Quiste hidatídico \\
\hline & Sarcoma pulmonar & & Ascaris \\
\hline
\end{tabular}

*potencialmente maligno.

Es importante destacar que los carcinoides bronquiales (adenomas bronquiales) son los tumores pulmonares con características histológicas benignos y son más frecuentes en los jóvenes. Se los con- sidera, no obstante, potencialmente malignos ya que pueden producir invasión local y diseminarse.

Radiológicamente, los NPS se clasifican como nódulos benignos o como nódulos indeterminados. Existen algunos criterios de benignidad mas seguros y específicos que otros. Estos criterios son la estabilidad en el tiempo y la presencia de calcificación. No obstante, luego de la evaluacion radiológica, la mayoría de los NPS terminan siendo categorizados como indeterminados.

La estabilidad de las dimensiones del tumor por un periodo mayor a dos años es otro indicador confiable de benignidad. Es fundamental intentar conseguir radiografías previas del paciente para fijarse si el nódulo en estudio ya se hallaba presente en aquellas y en caso afirmativo, comparar sus tamaños.

Una lesión que crece en semanas o meses es altamente sugestiva de malignidad.

\section{Calcificación y otras características morfológicas}

La presencia de una calcificación visible en la radiografía simple de tórax es un indicador confiable de benignidad. Las calcificaciones pueden ser de varios tipos: central o difusa, laminar o concéntrica (sugestiva de granuloma), en palomitas de maíz (sugestiva de hamartoma). En cambio, la presencia de una calcificación excéntrica debe sugerir malignidad, aunque rara vez un NPS maligno tiene calcificaciones.

La tomografía computada sin contraste ayuda a definir la anatomía del mediastino y mejora la sensibilidad para la detección de calcio como indicador de benignidad. En cuanto a la forma de los bordes, si bien es un hallazgo que posee poca sensibilidad, la presencia de un contorno nodular irregular o estriado sugiere malignidad. La tomografía computada también puede mostrar grasa, hallazgo que sugiere benignidad (hamartoma).

\section{¿Cómo estudiar un NPS?}

Es importante como primer paso asegurarse de que el hallazgo radiológico sea un verdadero nódulo pulmonar y no un artefacto de la técnica o una imagen prestada (un vaso visto de frente, un pezón, un nevo, etc.).

El paso siguiente consiste en buscar indicadores radiológicos de benignidad. Tanto las calcificaciones (visibles en la radiografía simple o en la tomografía computada), como la estabilidad del nódulo en un tiempo mayor a dos años (documentada con radiografías previas) definen benignidad.

Un nódulo calcificado (no incluye a la calcificación excéntrica) o una lesión estable, no merecen posterior evaluación.

Si el NPS resulta indeterminado (Lo más frecueñte) el siguiente paso consiste en evaluar el riesgo clínico de malignidad (edad, tamaño del NPS, tabaquismo, cancer previo). Estos factores definen la probabilidad previa a la biopsia (pretest) de que el tumor sea maligno como: baja, intermedia o alta. (Ver cuadro 2).

Las conductas posteriores son la observación, la biopsia (transtorácica o transbronquial) y la resección (por toracotomía o toracoscopía). La elección de las distintas alternativas se toma de acuerdo a que la probabilidad pretest haya sido baja, intermedia o alta respectivamente.

Cuadro 2: Coeficientes de probabilidad de malignidad de un nódulo pulmonar solitario en un varón según la regla probabilística de Cummings, Lilington y Richard 1 .

En la confección de esta regla de predicción estos autores consideraron que un nódulo pulmonar solitario tiene una probabilidad de ser maligno de 0.10 si se trata de un paciente ambulatorio (10\% de prevalencia de malignidad según datos provenientes de programas poblacionales de rastreo de tuberculosis realizados en los años cincuenta) y de 0.4 si se trata de un paciente internado (prevalencia 
de malignidad: $36 \%$ en la serie de Steele de 1963 y 44\% en la de Good y Wilson de 1958).

\begin{tabular}{cc}
\hline & CHANCE DE MALIGNIDAD EN ODDS \\
\hline Paciente internado & 0.7 \\
\hline
\end{tabular}

\begin{tabular}{|c|c|c|c|c|}
\hline Categorias & \multicolumn{3}{|c|}{ Característica clínica } & $\begin{array}{l}\text { Coeficiente de probabilidad } \\
\text { (CP) de malignidad }\end{array}$ \\
\hline \multirow[t]{6}{*}{ Edad del paciente } & \multicolumn{3}{|l|}{ Menor de 35 años } & 0.1 \\
\hline & \multicolumn{3}{|l|}{36 a 44 años } & 0.3 \\
\hline & \multicolumn{3}{|l|}{45 a 49 años } & 0.7 \\
\hline & \multicolumn{3}{|l|}{50 a 59 años } & 1.5 \\
\hline & \multicolumn{3}{|l|}{60 a 69 años } & 2.1 \\
\hline & \multicolumn{3}{|l|}{70 a 83 años } & 5.7 \\
\hline \multirow[t]{6}{*}{ Diámetro del nódulo } & \multicolumn{3}{|l|}{ Menor a $1.5 \mathrm{~cm}$} & 0.1 \\
\hline & \multicolumn{3}{|l|}{$1.5 \mathrm{a} 2.2 \mathrm{~cm}$} & 0.5 \\
\hline & \multicolumn{3}{|l|}{2.3 a $3.2 \mathrm{~cm}$} & 1.7 \\
\hline & \multicolumn{3}{|l|}{3.3 a $4.2 \mathrm{~cm}$} & 4.3 \\
\hline & \multicolumn{3}{|l|}{4.3 a $5.2 \mathrm{~cm}$} & 6.6 \\
\hline & \multicolumn{3}{|l|}{5.3 a $6.0 \mathrm{~cm}$} & 29.4 \\
\hline \multirow[t]{11}{*}{ Tabaquismo } & \multicolumn{3}{|l|}{ Nunca fumó } & 0.15 \\
\hline & \multicolumn{3}{|l|}{ Sólo pipa o cigarros } & 0.3 \\
\hline & \multicolumn{3}{|c|}{ Alguna vez fumó cigarrillos } & 1.5 \\
\hline & $\begin{array}{l}\text { Fumador actual de cigarrillos } \\
\text { o dejó de fumar hace menos } \\
\text { de nueve años }\end{array}$ & $\begin{array}{l}\text { Número de cigarrillos } \\
\text { fumado por día }\end{array}$ & 1 a 9 & 0.3 \\
\hline & & & 10 a 20 & 1 \\
\hline & & & 21 a 40 & 2 \\
\hline & & & 41 ó más & 3.9 \\
\hline & $\begin{array}{c}\text { Años desde que dejó } \\
\text { de fumar }\end{array}$ & & & 1.4 \\
\hline & & 4 & & 1 \\
\hline & & & & 0.5 \\
\hline & & & & 0.1 \\
\hline
\end{tabular}

La chance de malignidad (ODDs final o post-test) se obtiene luego de multiplicar los ODDs iniciales (0.1 ó 0.7 segŭn se trate, respectivamente de un paciente ambulatorio o internado) por los coeficientes de probabilidad de malignidad que corresponden a cada una de las categorías:

\section{ODDs final $2=0 \mathrm{DD}$ s inicial $\times$ CPedad $\times$ CPtamaño $\times$ CPtabaquismo}

La probabilidad final de cáncer se obtiene de la conversión de los ODDs finales a través de la fórmula:

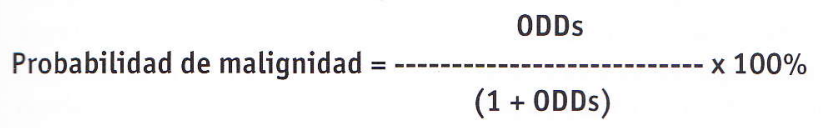

Ejemplo: a un paciente de 63 años que nunca fumó se le descubre un nódulo de $1.3 \mathrm{~cm}$ de diámetro en una radiografia de tórax que se hizo en un examen prequirúrgico por una cirugía ortopédica (hallux valgus).

Paciente ambulatorio $(O D D s=0.1)$,

edad 63 años $(C P=2.1)$,

tamaño $1.3 \mathrm{~cm}(C P=0.1)$,

nunca fumó $(C P=0.15)$.

ODDs final $=0.1 \times 2.1 \times 0.1 \times 0.15=0.00315$

\subsection{5}

Probabilidad de malignidad = - -

$(1+0.00315)$
(1) Cummings S, Lilington G, Richard R. Estimating probability of malignancy in solitary pulmonary nodules. A bayesian approach. Am Rev Respir Dis. 1986; 134:449-452).

(2) Es probable que la edad, el tamaño del nódulo y la historia de tabaquismo no sean variables independientes, por lo tanto es de esperar que este sistema de cálculo sobreestime la probabilidad de malignidad.

\section{Observación, resección o biopsia}

En un extremo del espectro de riesgo (probabilidad de malignidad baja) se encuentran los pacientes jóvenes (menores de 35 años) no fumadores, en quienes se puede seguir una conducta expectante de observación, con buena seguridad. Debe reevaluarse al paciente cada tres meses durante el primer año y cada seis meses durante el segundo. Si durante el seguimiento el nódulo impresiona agrandarse (aunque sea un aumento mínimo) se indica la resección del mismo.

En el otro extremo (probabilidad de malignidad alta) están los individuos mayores de 50 años que fuman y que se presentan con un nódulo grande (más de $2 \mathrm{~cm}$ ). En estos pacientes no cabe duda que la mejor conducta es la resección de la lesión.

Cuando el riesgo es intermedio la biopsia (transbronquial o transtorácica) es la mejor alternativa. La punción transtorácica con aguja fina es más sensible para diagnostico de malignidad que la transbronquial (sobre todo en los nódulos periféricos y grandes) pero 
presenta más riesgo de complicaciones (neumotorax: 11 a 24\%; hemóptisis menor: 10\%) y permite sólo un análisis citológico (bajo rédito diagnóstico para las patologías benignas).

La biopsia transbronquial tiene menos efectos adversos (mortalidad: 0.2\%; neumotórax: $5.5 \%$; hemorragia de más de $50 \mathrm{ml}: 1$ a $4 \%$ ) pero también una menor sensibilidad diagnóstica. La sensibilidad aumenta con el lavado y el cepillado bronquial.

El hallazgo de una patología benigna específica excluye al cancer en forma efectiva (evita la resección) salvo en los pacientes que tienen una muy elevada probabilidad de malignidad previa a la biopsia.

La toracoscopía tiene utilidad en la biopsia o en la resección de nódulos periféricos (visibles en la inspección).

La toracotomía es la prueba de referencia ("gold-standard") para el diagnóstico, estando indicada en los nódulos indeterminados con alto riesgo clínico de malignidad, en los nódulos que crecen y luego de una biopsia con un resultado indeterminado en un paciente con alto riesgo.

\section{Consulta con el especialista y recomendaciones}

El grupo de pacientes que genera mayores dudas diagnósticas es el de aquellos individuos con NPS indeterminados y probabilidad intermedia de malignidad, especialmente si ya existe una biopsia con un resultado no diagnóstico. En estas situaciones es difícil decidir entre las tres alternativas posibles: observación del paciente, nueva biopsia o resección de la lesión. Son éstas, las situaciones clínicas de más difícil resolución y en las cuales se requiere el consejo del especialista.

El principal esfuerzo del médico debe orientarse a evitar el hábito de fumar. Aunque la evidencia acumulada hasta hoy es controvertida; no está recomendado (ni siquiera en fumadores) el rastreo radiológico del cancer de pulmón, ya que no parece modificar la historia natural de la enfermedad.

\section{Dr. Eduardo Abbatte \\ Clínica médica \\ CEMIC}

\section{Bibliografía recomendada}

Cummings S, Lilington G, Richard R. Estimating probability of malignancy in solitary pulmonary nodules. A bayesian approach. Am Rev Respir Dis. 1986; 134:449-452.

Melamed M, Flehinger B, Zaman M, et al. Screening for Early Lung Cancer. Results of the Memorial Sloan-Kettering Study in New York. Chest 1984: 86:44-53.

Midthun D, Swensen S, Jett J. Approach to the Solitary Pulmonary Nodule. Mayo Clin Proc 1993: 68: 378-385.

(Revisión completa y práctica para el médico generalista).

Minna J. Neoplasms of the Lung. En: Fauci A, Braunwald E, Isselbacher K, Wilson J, Martin J, Kasper D, et al. Harrison's Principles of Internal Medicine. 14th edit.Mc Graw-Hill Companies, Inc. 1998. P. 552-562.

Pugatch R. Radiologic Evaluation in Chest Malignancies. A Review of Imaging Modalities. Chest 1995:107: 294S-297S.

Tape T. Solitary Pulmonary Nodule. En: Panzer R, Black E, Griner P, editors. Diagnostic strategies for common medical problems. 2 nd edit. 1991. P 270-278. Este articulo ofrece un analisis detallado acerca del rédito de cada examen complementario y ayuda a calcular, mediante tablas, la probabilidad pretest de malignidad. 